La Revue

des Droits

de l'Homme

\section{La Revue des droits de l'homme}

Revue du Centre de recherches et d'études sur les droits fondamentaux

$1 \mid 2012$

Revue des droits de l'homme $-\mathrm{N}^{\circ} 1$

\title{
Section 1. Standards jurisprudentiels et contrôle de l'obligation étatique
}

Des contrôles à géométrie variable

Isabelle Boucobza et David Robitaille

\section{OpenEdition}

Journals

Édition électronique

URL : http://journals.openedition.org/revdh/142

DOI : $10.4000 /$ revdh. 142

ISSN : 2264-119X

Éditeur

Centre de recherches et d'études sur les droits fondamentaux

Édition imprimée

Date de publication : 1 juin 2012

Pagination : 298-319

Référence électronique

Isabelle Boucobza et David Robitaille, "Section 1. Standards jurisprudentiels et contrôle de l'obligation étatique », La Revue des droits de l'homme [En ligne], 1 | 2012, mis en ligne le 30 juin 2012, consulté le 08 juillet 2020. URL : http://journals.openedition.org/revdh/142 ; DOI : https://doi.org/10.4000/revdh. 142 


\title{
CHAPITRE 1 - DROIT COMPARE
}

\author{
SECTION 1 \\ STANDARDS JURISPRUDENTIELS ET CONTROLE \\ DE L'OBLIGATION ETATIQUE EN DROIT COMPARE : \\ UNE GEOMETRIE VARIABLE
}

\section{ISABELLE BOUCOBZA \& DAVID ROBITAILLE}

L'étude comparative menée dans le cadre du programme de recherche sur la justiciabilité des droits sociaux a permis d'identifier des techniques souvent très proches qui sanctionnent le respect par le législateur étatique de "standards jurisprudentiels". Ces standards, entendus ici comme des définitions matérielles des obligations étatiques, que les juges tirent des textes constitutionnellement consacrés, servent ensuite de fondement pour évaluer les actions et les omissions législatives.

Cette contribution propose de présenter la façon dont les juges parviennent à la formulation de ces normes, dont ils organisent aussi le contrôle à partir de méthodes ou de formes de décisions dont certaines ont été spécifiquement inventées pour la protection des droits sociaux. Il faut cependant noter que ces mêmes techniques peuvent parfois servir l'effet inverse de non protection ou d'abaissement du niveau de protection; elles sont alors associées soit avec des arguments spécifiques qui servent à justifier la non protection ou l'abaissement du niveau de garantie, soit avec des arguments qui peuvent sembler identiques comme le caractère "raisonnable" d'un balancement entre deux principes.

La formulation des standards jurisprudentiels apparaissant en même temps que la mise en œuvre des techniques juridictionnelles qui visent à les protéger, il est apparu opportun de distinguer entre les techniques juridictionnelles elles-mêmes (I) et les arguments qui sont utilisés pour les justifier(II). 


\section{Les techniques juridictionnelles de protection des droits sociaux}

On concentrera ici l'attention sur deux aspects de la question des techniques à travers lesquelles les juges formulent, ou au contraire se refusent à formuler, des standards jurisprudentiels : d'abord le recensement des techniques elles-mêmes (A), ensuite l'intensité des contrôles (B).

\section{A. Les techniques recensées}

1) La mise en place de "seuils" de protection: des jurisprudences ambigües

\section{a. Les équivoques sur l'existence d'une "obligation de non rétrogression"}

"Encliquetage» ou "standill» dans le droit belge, jurisprudence du "cliquet" dans le droit français, ces expressions ont toute en commun avoir été forgées par la doctrine pour commenter et interpréter une jurisprudence qui a généré de nombreuses équivoques. En effet, lorsqu'il est compris comme un principe d'interdiction de régression sociale, autrement dit comme la reconnaissance d'acquis sociaux irréversibles, ou encore de droits intangibles, ce principe ne reçoit aucune confirmation dans les décisions de juridictions constitutionnelles ${ }^{727}$.

Cette interprétation optimiste en France de la décision du Conseil constitutionnel de 1984 "Entreprise de presse" qui pose que "le pouvoir législatif ne peut réglementer l'exercice de la liberté de communication des pensées que dans le but de le rendre plus effectif " a été à la fois confirmée dans son principe - celui de l'obligation faite au législateur de rendre les droits effectifs - et en même temps infirmée puisque les décisions postérieures n'ont pas exigé que le législateur garantisse un droit à un toujours mieux (ou à plus d'effectivité), ni même au maintien de l'acte législatif existant. La formule issue de la décision de 1986 "régime de Presse où le Conseil constitutionnel pose en effet que «si le législateur peut librement abroger ou modifier des dispositions législatives antérieures, l'exercice de ce pouvoir ne saurait aboutir à priver de " garanties légales des exigences de caractère constitutionnel " ${ }^{728}$ semble réduire l'obligation du législateur au maintien d’une réglementation, quel que soit son contenu ${ }^{729}$. Il paraît difficile d'y voir une sorte de constitutionnalisation de toute

727 V. Isabelle HACHEZ, « La reconnaissance jurisprudentielle du principe de Standtill en droit belge ", in Julia ILIOPOULOS-STRANGAS et Theunis ROUX, dir., Perspectives nationales et internationales des droits sociaux, Athène/Ant. N. Sakkoulas, Bruxelles/Bruylant, 2008, pp. 200201.

728 CC, n 86-210 DC, 29 juillet 1986, Régime de la presse.

$729 \mathrm{Il}$ s'agit également de l'obligation minimale à laquelle est assujetti le gouvernement canadien en vertu des droits économiques et sociaux reconnus dans la Charte des droits et libertés de la personne du Québec (Gosselin c. Québec (Procureur général), [2002] 4 R.C.S. 429 (Canada, C.S.) [Gosselin (Cour suprême)], par. 87 à 96. 
avancée dans la réalisation des droits sociaux ou alors faudrait-il peut être parler plus justement d'une cristallisation constitutionnelle relative.

Formulée par les textes constitutionnels eux-mêmes, l'idée d'une obligation de non rétrogression peut alors s'entendre dans deux sens: soit comme une interdiction de révision constitutionnelle, le droit ne serait pas même attaquable par le législateur constitutionnel ; soit comme la formulation d'une interdiction de toucher à une définition " minimale" ou " essentielle " du droit ${ }^{730}$ ou autrement dit, à la définition d'un contenu prioritaire des droits sociaux, qui constitue effectivement une technique de mise en œuvre juridictionnelle de seuils de protection, formellement identifiable dans différentes jurisprudences constitutionnelles, mais non dénuée d'ambiguïtés.

\section{b. La définition d'un premier niveau de l'obligation de protection: le contenu minimum essentiel d'un droit}

Le Comité des droits économiques, sociaux et culturels des Nations Unies estime que les États parties au PIDESC ont l'obligation fondamentale de garantir le niveau minimal de tous les droits reconnus par le traité de façon à assurer à tous les besoins essentiels à une vie digne ${ }^{731}$.

Ainsi, le CoDESC a par exemple déterminé le contenu minimum du droit $\grave{a}$ un niveau de vie suffisant reconnu au paragraphe $11 \mathrm{du}$ PIDESC. Cette disposition précise que le droit à un niveau de vie suffisant protège le droit à un logement décent, lequel doit notamment comprendre l'eau potable, l'électricité, les installations sanitaires et un espace suffisant 732 , à un coût correspondant à une fraction raisonnable du revenu de ses habitants ${ }^{733}$, ainsi que le droit à une nourriture saine et propre à la consommation, à des prix raisonnables ${ }^{734}$.

En droit national, la formulation d'un «contenu» «minimal» ou "essentiel», parfois également exprimé comme celle d'un "noyau dur» d'un droit, apparaît comme une limite à la modification législative. Or, cette formule est ambigüe : dans la mesure où elle s'applique à des dispositions qui impliquent la mise en œuvre d'un pouvoir discrétionnaire, c'est-à-dire d'un choix de moyens pour le législateur afin d'atteindre une fin plus ou moins définie, elle peut donner lieu à plusieurs interprétations qui seront vues comme étant plus ou moins favorables à la protection des droits sociaux. Ainsi, elle peut être entendue :

- d'un point de vue purement formel comme une simple habilitation législative, c'est l'idée déjà évoquée d'un traitement législatif quel que soit son

\footnotetext{
730 Ainsi, l'affirmation répétée dans la jurisprudence constitutionnelle italienne du caractère " inviolable» des droits fondamentaux présente suffisamment d'ambigüités pour être comprises dans les deux sens et justifier ces deux formes de protections (v. infra).

731 V. supra, Sophie GROSBON.

732 Ibid., par. 8 (b), (d) et (e).

733 Ibid., par. 8(c).

734 Observation générale 12, art. 8, 13.
} 
contenu. Le législateur doit légiférer, au-delà de cette obligation, il est absolument libre.

- d'un point de vue formel et matériel : elle combine alors l'habilitation législative et la définition d'un contenu prioritaire des droits sociaux qui sera plus ou moins contraignant et plus ou moins protecteur.

On trouve une illustration de la conception purement formelle de la notion dans le droit canadien, et plus précisément dans l'interprétation donnée à la Charte québécoise ${ }^{735}$. Les droits économiques et sociaux reconnus dans la Charte québécoise imposeraient au gouvernement l'obligation de les rendre effectifs par l'adoption, par exemple, d'un régime législatif. L'« effectivité " dont il est ici question s'entend, évidemment, d'effectivité minimale ou purement formelle dans la mesure où les tribunaux n'exerceront aucun contrôle sur la suffisance de la loi, leur pouvoir se limitant à constater l'existence d'un tel régime ou, à défaut, a déclarer que l'omission du législateur à mettre un droit social donné en œuvre ne respecte pas la Charte. Dans ce dernier cas, la constatation de la Cour demeurera purement formelle puisque le juge ne dispose pas du pouvoir de contraindre le gouvernement à faire quoi que ce soit pour y remédier.

La conception formelle et matérielle quant à elle prend forme dans l'expression de standards, qui pourront aller vers le haut mais qui ne pourront pas tendre vers le bas. Dans cette seconde hypothèse, l'accent est mis sur la définition juridictionnelle du droit : il s'agit ni plus ni moins pour le juge que de formuler explicitement les obligations "minimales" «essentielles" qui découleraient de la consécration constitutionnelle d'un droit social. On touche ici une question très controversée puisque cette technique apparaît comme un remède juridictionnel au caractère insuffisamment précis ou vague de la formulation d'un droit social. On sait en effet toutes les conséquences que la doctrine a traditionnellement tiré de cette imprécision pour justifier la faible justiciabilité des droits sociaux, l'imprécision, le caractère programmatique mettant en cause jusqu'à à l'existence même d'un droit justement parce que sa définition dépendrait exclusivement de la volonté discrétionnaire du législateur. C'est d'ailleurs l'une des raisons pour lesquelles la Cour constitutionnelle sudafricaine, dans l'interprétation des droits économiques et sociaux reconnus par la Constitution de 1996, a refusé de transposer dans le droit interne le concept de notion minimal ou essentiel développé en droit international. La Cour estime que le contenu des droits sociaux est trop variable ou indéterminé pour faire l'objet d'une définition précise, et que tel n'est pas son rôle.

En formulant une définition, l'interprète authentique "prend le pouvoir " et donne au droit un contenu, c'est-à-dire qu'il tranche la question controversée.

735 Canada, C.S., Gosselin, précit., par. 86-88. 
Il dit ce que dit le droit social736, il énonce au législateur les obligations auxquelles son pouvoir discrétionnaire ne peut dès lors déroger en explicitant les finalités qu'il doit nécessairement atteindre et qui détermineront avec plus ou moins d'amplitude l'éventail des moyens à sa disposition.

La jurisprudence de la Cour constitutionnelle italienne en offre de nombreuses illustrations: il faut noter que la Constitution italienne comporte un catalogue important de droits sociaux et la Cour a fait preuve d'un activisme marqué pour assurer leur protection. Ceci explique que la question d'une mesure minimale des droits sociaux n'apparaisse qu'au début des années 1990 dans sa jurisprudence. Elle a d'ailleurs été critiquée par certains auteurs en Italie car elle a été vue comme une technique d'abaissement du niveau de protection de certains droits sociaux, comme une modalité retour en arrière, expression d'un self restraint du juge ${ }^{737}$. La définition de ce contenu minimum essentiel des droits sociaux coïncide en effet avec une période de compression des ressources financières de l'Etat et de pression sur les critères d'équilibre budgétaire, notamment en liaison avec l'engagement de l'Italie dans l'Union européenne. Dans la décision 992/1988, la Cour déclare inconstitutionnelle une disposition législative qui ne prévoyait pas le remboursement par le service national de santé des frais coûteux de diagnostic spécialisé effectué par des laboratoires privés non conventionnés mais qui étaient les seuls à disposer du matériel nécessaire à la réalisation d'examens indispensables pour le patient. En censurant cette disposition, elle signifiait donc au législateur qu'il n'était pas libre de déterminer le contenu de ce droit et qu'en l'espèce, les mesures adoptées n'étaient pas suffisantes pour respecter le droit à des prestations sanitaires. Pour la Cour, l'article 32 de la constitution qui énonce le droit à la protection de la santé contient celui de pouvoir accéder à des prestations indispensables dès lors que la technologie le permet. Selon une jurisprudence désormais abondante et constante, le juge constitutionnel italien a établi qu'aucun droit fondamental ne peut être compressé ou limité au point que son contenu minimum essentiel en soit compromis ou risque de l'être. ${ }^{738}$

Il faut encore signaler qu'aujourd'hui la notion de contenu minimal essentiel des droits sociaux semble toujours plus liée à la question des disponibilités financières dans une conjoncture économique défavorable. Or, ce

\footnotetext{
736 Bien entendu, il faudra s’interroger sur la façon dont les juges justifient cette interprétation non seulement en sollicitant des arguments tels que nature juridique, intention du constituant etc...mais également en prétendant que le droit est doté d'un contenu propre prédéterminé (voir infra II) Pour une analyse plus approfondie, v. David ROBITAILLE, "L'interprétation en théorie du droit comparée : entre la lettre et l'esprit. Discussion autour d'auteurs américains, anglais, belges, canadiens et français ", Revue de la recherche juridique, Droit prospectif, 2007, vol. 119, p. 1145 ; v. également Isabelle BOUCOBZA, La fonction juridictionnelle, contribution à une analyse des débats doctrinaux en France et en Italie, Dalloz, Collection nouvelle bibliothèque de thèses, Paris, 2005.

737 Carmela SALAZAR, Dal riconoscimento alla garanzia dei diritti sociali. Orientamenti e tecniche decisorie della Corte costituzionale a confronto, Torino, 2000, p. 131.

738 V. parmi d'autres les décisions 455/1990, 247/1992, 218-304/1994, 416/1995, 27-185-267/1998, 309/1999, 509/2000, 432/2005.
} 
balancement entre ressources financières disponibles et droit donne des résultats contraires au principe d'interdépendance et d'indivisibilité de tous les droits et libertés, puisque il emporte une hiérarchisation entre les droits sociaux. Carmela Salazar ${ }^{739}$ souligne ainsi que, dans la jurisprudence italienne, la technique du contrôle minimum aurait donné au droit à la santé une position de droit préféré par rapport aux droits des travailleurs.

Selon certains, les contraintes budgétaires ne devraient pas entrer en jeu à l'étape de la "détermination" du contenu essentiel des droits sociaux mais plutôt à celle consistant à vérifier si le gouvernement pouvait raisonnablement manquer à son obligation ${ }^{740}$. Toutefois, l'exemple italien montre que c'est précisément au moment où la Cour s'est montrée plus sensible à l'argument de la compétence budgétaire du Parlement et de la disponibilité des ressources financières qu'elle a fait appel à la notion.

Enfin, à la pluralité des lectures possibles des décisions on doit ajouter des difficultés d'ordre terminologique: d'abord, il faut souligner que les expressions de "continu minimal", de "contenu essentiel» ou de "noyau essentiel» ne sont pas dépourvues d'ambiguïtés. Notamment on peut se demander si les notions de "contenu essentiel » et de " contenu minimal " sont synonymes. Ensuite, la terminologie n'est pas figée et de nouvelles expressions apparaissent: " exigences minimum de protection » 741 , "noyau indéfectible des garanties $»^{742}$.

2) Le recours à d'autres droits ou l'interprétation "perméable " des droits à la vie, à la sécurité ou à l'égalité

Une autre technique fréquemment utilisée est le recours à d'autres droits et libertés fondamentaux qui, interprétés largement, pourraient inclure certains droits économiques et sociaux ${ }^{743}$. Au Canada, comme la constitution ne garantit aucun droit socioéconomique, les articles 7 et 15 de la Charte canadienne, qui reconnaissent respectivement le droit à la vie et à la sécurité et le droit à l'égalité, ont été mobilisés afin de faire reconnaître, notamment, le droit à un niveau de vie suffisant ainsi que le droit à l'égalité réelle. Il a ainsi été soulevé que pour bénéficier d'une sécurité, d'une vie ou d'une égalité véritable, un certain niveau de protection sociale était nécessaire, sans quoi ces valeurs ou

739 Carmela SALAZAR, Dal riconoscimento alla garanzia dei diritti sociali. Orientamenti e tecniche

decisorie della Corte costituzionale a confronto, précit., p. 3.

740 David BILCHITZ, "Towards a Reasonable Approach to the Minimum Core: Laying the Foundations for Future Socio-economic Rights Jurisprudence», S.A.J.H.R., 2003 (19), Johannesburg, 1, pp.22-23 ; "Giving Socio-economic Rights Teeth : The Minimum Core and its Importance", S. African L.J., 2002 (119), 484, p. 496; Laurence GAY, Les "droits-créances" constitutionnels, Bruxelles, Éditions Bruylant, 2007, p. 654.

741 V. arrêt n $202 / 2008$.

742 V. arrêt n 80 de 2010.

${ }^{743}$ Pour de plus amples détails, v. Diane ROMAN, infra. 
droits seraient superficiels ou incomplets ${ }^{744}$. Les tribunaux canadiens se sont cependant montrés très hésitants à se prononcer sur les choix socioéconomiques du gouvernement et à intervenir dans un domaine qui, selon eux, relève de la politique ${ }^{745}$. Il faut reconnaître également que la Charte canadienne n'est pas la plus progressiste des constitutions et que son objectif n'était pas tant d'assurer une plus grande justice sociale mais plutôt la préservation de l'autonomie et de la liberté individuelles (formelles). La technique a connu un plus grand succès dans la jurisprudence de la Cour suprême indienne, cette dernière reconnaissant, sur le fondement d'une conception globale du droit à la vie, plusieurs droits économiques et sociaux ${ }^{746}$. Pourtant, la Constitution indienne contient un chapitre distinct consacrant des principes socioéconomiques que le constituant a expressément prévu comme étant non-justiciables. Il est probable que la Cour suprême ait néanmoins eu recours à une interprétation large du droit à la vie - poussant ainsi plus loin les frontières de la séparation des pouvoirs judiciaire et législatif -, afin de pousser le gouvernement à agir pour faire diminuer la grande pauvreté qui affecte plusieurs citoyens. Il a en effet été remarqué que la jurisprudence la plus progressiste de la Cour a été développée dans les années 1990, alors que le gouvernement indien mettait sur pieds des politiques de libéralisation des marchés et d'ajustements structurels ayant pour effet d'augmenter la pauvreté des personnes les plus pauvres ${ }^{747}$. La réussite de cette technique est donc tributaire de la façon dont le juge conçoit les droits à la vie, à la sécurité ou à l'égalité (conception substantielle vs. formelle de la vie, de la sécurité ou de l'égalité) et du contexte dans lequel est interprétée la norme constitutionnelle.

Dans certaines décisions, la Cour constitutionnelle italienne associe un droit social avec un droit ou principe tel que celui de la dignité humaine ${ }^{748}$. Mais le droit « classique " auquel la Cour fait le plus souvent appel, en le combinant avec les dispositions qui protègent les droits sociaux, est sans conteste le droit à

\footnotetext{
744 V. notamment les motifs dissidents de la juge ARBOUR dans l'arrêt Gosselin (Canada, C.S.), supra, par. 307-394.

745 Pour une analyse plus détaillée de la jurisprudence canadienne, v. notamment Jamie CAMERON, " Positive Obligations Under Sections 15 and 7 of the Charter: A Comment on Gosselin v. Québec ", Sup. Ct. L. Rev. (2d), 2003 (20), Toronto, 65 ; Angus GIBBON, "Social Rights, Money Matters and Institutional Capacity ", Revue nationale de droit constitutionnel, 2003 (14), Scarborough (Canada), 353 ; Martha JACKMAN, " Charter Equality at Twenty: Reflections of a Card-carrying Member of the Court Party ", Revue nationale de droit constitutionnel, 2006 (20), Scarborough (Canada), 115 ; Martha JACKMAN, " Poor Rights : Using the Charter to Support Social Welfare Claims ", Queen's L. J., 1993 (19), Kingston (Canada), 65 ; Natasha KIM et Tina PIPER, "Case Comment. Gosselin v. Quebec : Back to the Poorhouse... ", Revue de droit de McGill, 2003-2004 (48), Montréal, 749 ; David ROBITAILLE, " Nonuniversalité, illégitimité et sur-complexité des droits économiques et sociaux : des préoccupations légitimes mais hypertrophiées. Regard sur la jurisprudence canadienne et sud-africaine ", (2008) ${ }^{53}$ Revue de droit de McGill 243.

746 Pour de plus amples détails sur la jurisprudence indienne, v. Diane ROMAN, infra et David ROBITAILLE, supra.

747 V. David ROBITAILLE, "L'influence du contexte économique et idéologique sur la conception de l'être humain par le droit et le juge constitutionnels : les cas canadien, indien et sud-africain ", Revue canadienne droit et société /Canadian Journal of Law and Society, à paraître. 748 Ex. Arrêt n 252/2001.
} 
l'égalité, consacré dans l'article 3 de la constitution. Sur son fondement, deux types de contrôle se sont développés : le premier est présenté comme un contrôle de "rationalité " ou de " cohérence " permettant de sanctionner une disparité de traitement. Sa particularité résiderait dans son caractère "ternaire " ${ }^{749}$ : la disposition constitutionnelle dont on dénonce la violation est évidemment l'art. 3, mais la violation elle-même dériverait de la disparité de traitement réalisée par la loi dont la constitutionnalité est discutée par rapport à ce qui est fait par une autre loi utilisée comme point de comparaison (tertium comparationis) pour une catégorie de sujets semblables. La Cour apprécie alors le caractère "raisonnable", "rationnel" ou " cohérent" - ces différents termes ne faisant pas l'objet d'un usage rigoureux de la part du juge - de la différence de traitement. Ce type de jugement en matière de droits sociaux a donné lieu à des sentences très incisives à l'égard du pouvoir du législateur puisqu'elles consistent en une addition de prestation: lorsqu'il juge une différence de traitement discriminatoire - la loi a traité de façon différente des personnes qui se trouvaient dans une situation identique à celles qui étaient protégées par l'autre loi, le juge constitutionnel n'a pas hésité à étendre le bénéfice d'une prestation à une catégorie qui en était illégitimement exclue ${ }^{750}$. Cependant le recours au principe d'égalité peut également conduire au retrait d'une prestation. Ainsi, la Cour, au lieu d'intervenir pour étendre le bénéfice d'un dispositif à d'autres catégories de sujet, a, dans certains cas, décidé de supprimer un privilège jugé irraisonnable, réalisant ainsi un nivellement vers le bas $^{751}$.

Le second type de contrôle est présenté comme un contrôle de "raisonnabilité » dont la particularité serait d'être binaire, il confronte la loi au principe d'égalité -, et consisterait essentiellement en une sorte d'examen des raisons d'être de la loi, de son caractère non arbitraire, au sens où les intérêts qu'elle protège sont raisonnablement compatibles avec les valeurs de la constitution ou qu'ils sont le fruit d'un « balancement raisonnable » entre elles.

Malheureusement, la distinction théorique et doctrinale entre ces deux types de contrôle se heurte à la réalité jurisprudentielle où il est bien difficile de les isoler. La Cour Sud africaine pratique également ces jugements de raisonnabilité qui méritent donc des développements spécifiques.

\footnotetext{
749 Alfonso CELOTTO, «Egualianza e ragionevolezza nella giurisprudenza costituzionale italiana ", Biblioteca juridica virtual del instituto de investigaciones juridicas de la UNAM, www.bibliojuridica.org/libros/6/2557/11.pdf.

${ }^{750}$ Parmi tant d'autres, v. par exemple l'arrêt de $1974 \mathrm{n}^{\circ} 230$, pour plus d'illustrations présentées en français, v. Jean-Jacques PARDINI, Le juge constitutionnel et le fait en Italie, Economica, 2001 751 Arrêt n 421/1995, Giur. Cost. 1995, p. 3237 et s.
} 
3) Les "jugements de raisonnabilité": des contrôles de "l'excès de pouvoir législatif "?

\section{a. Dans la jurisprudence de la Cour constitutionnelle italienne}

Du côté de la Consulta, le principe d'égalité s'est progressivement transformé en principe de raisonnabilité, c'est à dire en une sorte de "clause générale " qui permet la limitation des choix législatifs à partir de l'examen de la légalité, de la cohérence, de la proportionnalité de la mesure retenue dans la loi au regard des objectifs de celle-ci mais également des valeurs défendues par le système juridique. Cette diffusion du contrôle de raisonnabilité qui n'est donc pas spécifique aux droits sociaux ${ }^{752}$ pose souvent des difficultés de lecture : d'abord parce que cette jurisprudence serait devenue par sa densité tout à fait "ingérable "; ensuite parce que la systématisation doctrinale, elle-même victime du succès de la " raisonnabilité ", fait peut être courir le risque de mettre de l'ordre là où il n'y en a pas.

Ces précautions prises, il n'en demeure pas moins possible de percevoir que, par cette technique, la Cour procède ni plus ni moins à ce que l'on coutume d'appeler en droit administratif un contrôle de "l'excès de pouvoir", en l'occurrence, législatif.

Ainsi, dans la décision n. 421 de 1991, la Cour déclare l'inconstitutionnalité de la disposition qui prévoit, qu'en cas de transformation du contrat de travail d'un temps plein à un temps partiel et vice versa, le montant de l'indemnité de fin de contrat est calculée sur la base de la rétribution correspondant aux douze derniers mois de service. Cette disposition est jugée contraire à l'article 3 de la Constitution tant du point de vue du principe d'égalité que de celui du principe de rationalité. Sur le premier point, elle traite en effet de façon identique des situations différentes, ce qui crée un désavantage pour l'individu qui a travaillé à temps partiel seulement dans sa dernière année de contrat et, en même temps, une situation avantageuse mais injustifiée pour celui qui a donné un service à temps complet dans sa dernière année. Sur le second point, c'est parce que l'indemnité est liquidée sur la base d'une rétribution réduite en cas de travail à temps partiel, même pour les années de services effectuées à temps plein, sans tenir compte que pour ces années, l'employeur et le salarié ont versé une contribution proportionnelle à l'entière rémunération, que la loi est censurée. La démarche de la Cour ici est parfaitement bien illustrée: elle sanctionne la violation de l'égalitéraisonnabilité, la norme en cause introduit un traitement égal de situations différentes, mais elle exprime également un jugement sur la rationalité intrinsèque de la disposition.

Le juge impose ainsi au législateur une obligation de cohérence qui s'applique à la logique interne des textes contrôlés mais également aux secteurs

\footnotetext{
752 Andrea MORRONE, Il custode della ragionevolezza, Milano, ed. Giuffrè, 2001.
} 
juridiques auxquels ils appartiennent. On peut aussi y voir une exigence de conformité à des principes de justice et d'équité qui fonctionnent comme critères de jugement de la "raisonnabilité extrinsèque " : le juge constitutionnel observe la conformité du dispositif avec les raisons et les valeurs qui fondent l'entier système constitutionnel.

Cette dernière déclinaison de la raisonnabilité peut être illustrée avec une décision de la Cour constitutionnelle relative aux discriminations fondées sur la citoyenneté pour la jouissance de prestations sociales : une disposition de la loi régionale lombarde est jugée contraire au principe d'égalité. Elle garantissait la circulation gratuite dans les transports régionaux, non à toutes les personnes résidentes en Lombardie et qui souffrent d'une invalidité égale à $100 \%$, mais seulement à celles qui ont la nationalité italienne. La Région justifie la discrimination en retenant que les prestations de transport gratuites mises en place allaient bien au-delà des niveaux essentiels de prestations et qu'elles constituaient en cela des prestations "facultatives" qui devaient respecter les exigences de l'équilibre budgétaire. Si la Cour estime correcte dans son principe cette ligne de défense, elle la juge tout à fait hors de propos en ce qui concerne la disposition en cause.

"Le fait que la Région ait introduit un régime de faveur qui sans aucun doute excède les limites de "l'essentiel" (...) n'exclut en aucune manière que les choix liés à la désignation des bénéficiaires - qu’il faut nécessairement circonscrire en raison du caractère limité des ressources financières - doivent être opérés, toujours et en tout état de cause, dans le respect du principe de raisonnabilité ; en effet, le législateur étatique ou régional ne peut introduire des régimes différenciés, concernant le traitement à réserver aux individus, qu'en présence d'une "cause" normative non manifestement irrationnelle ou arbitraire ». Si la Cour juge que le critère de résidence n'est pas déraisonnable dans le cadre d'une réglementation régionale, en revanche elle rejette le critère de la citoyenneté «condition ultérieure, peu pertinente et incohérente par rapport à une mesure sociale qui fait des invalides à 100\% la catégorie des bénéficiaires". Soulignant que sa jurisprudence a rappelé à plusieurs reprises au législateur le principe fondamental et indérogeable de non discrimination sur la base de la citoyenneté à l'égard des prestations sociales quelles qu'elles soient, la Cour fait de ce principe "le modèle sur le fondement duquel elle calibre son jugement de raisonnabilité » et précise : "tout choix du législateur qui introduirait des régimes dérogatoires à ce principe doit présenter dans sa structure normative même une spécifique transparente et rationnelle 'cause justificatrice' en mesure " d'expliquer " sur le plan constitutionnel, les "raisons posées au fondement de la dérogation" (432/2005). Enfin, cette technique du jugement de raisonnabilité se présente également comme une méthode de balancement entre des intérêts, des droits et des valeurs protégées par la constitution. Parmi ces valeurs, en matière de droits sociaux, on trouve notamment, dans une jurisprudence de 1990 ( $\mathrm{n}^{\circ}$ 260), celle de "l'équilibre financier. 
Ces divers jugements de raisonnabilité conduisent à des résultats très différents du point de vue de leur intensité. Comme le contrôle de l'excès de pouvoir en droit administratif, l'intensité variable auquel il peut donner lieu prend toute son importance en matière de droits sociaux. En effet, le contrôle de raisonnabilité, lorsqu'il porte sur l'exercice du pouvoir discrétionnaire du législateur, peut conduire le juge à exercer un contrôle en profondeur des décisions du législateur, si pénétrant qu'il peut aboutir à la substitution de ses choix.

\section{b. Dans la jurisprudence de la Cour constitutionnelle sud-africaine}

Dans l'arrêt Government of the Republic of South Africa c. Grootboom ${ }^{753}$, la Cour constitutionnelle sud-africaine a élaboré ce que plusieurs considèrent comme une approche inspirée du droit administratif ${ }^{754}$, en vertu de laquelle le rôle du tribunal est de vérifier si les politiques publiques sont cohérentes, rationnelles et suffisamment équilibrées entre la poursuite d'objectifs - et la satisfaction corollaires de besoins - à court, à moyen et à long terme ${ }^{755}$. Cette norme de contrôle est en soi très floue et c'est pourquoi la Cour a tenté de la préciser en spécifiant que des politiques qui omettent de tenir compte des besoins prioritaires et de la grande pauvreté dans laquelle vivent certains groupes de personnes seront souvent jugées déraisonnables ${ }^{756}$.

Contrairement au concept de noyau minimal que la Cour a rejeté ${ }^{757}$, cette norme de raisonnabilité n'oblige pas l'État à satisfaire les besoins essentiels de tous avant de viser la réalisation d'autres objectifs à moyen ou à long terme. S’il est par ailleurs difficile de déterminer de manière précise et prévisible la mesure dans laquelle une politique doit tenir compte de la situation des plus défavorisés pour être raisonnable, la Cour indique néanmoins que des programmes qui omettent de prioriser les besoins minimaux d'un trop grand nombre de personnes seront jugés inconstitutionnels ${ }^{758}$. C'est sur la base de ce test que la Cour constitutionnelle jugea arbitraire une politique en matière de logement dont les ressources étaient concentrées vers la construction de logements sociaux, un objectif à moyen sinon à long terme ${ }^{759}$, et qui ne prévoyait aucune mesure pour alléger la misère des demandeurs qui, occupant des bidonvilles, vivaient dans des conditions extrêmement difficiles ${ }^{760}$. C'est également en fonction de cette norme que la Cour jugea arbitraire une politique en vertu de laquelle l'État limitait de façon importante l'accessibilité publique à un médicament susceptible de prévenir la transmission du VIH entre une mère et

\footnotetext{
753 [2001] 1 S. Afr. L.R. 46 (S. Afr. Const. Ct.) (ci-après Grootboom).

754 V. not. Alana KLEIN, « Judging as Nudging: New Governance Approaches for the Enforcement of Constitutional Social and Economic Rights ", Colum. Hum. Rts. L. Rev., 2007-2008 (39), New York, 351, pp. 374-385.

755 Grootboom, supra, par. 43.

756 Ibid., par. 44.

757 Ibid., par. 32, 35 et 37 .

758 Ibid., par. 43, 52-69.

759 Ibid., par. 51-52, 63 et 64 .

${ }^{760}$ Ibid., par. 7, 52-69.
} 
son enfant et qui était offert gratuitement par le fabricant pour une période de cinq ans ${ }^{761}$.

\section{B. Des techniques de contrôle à intensité variable}

Comme nous venons de le voir, la Cour constitutionnelle sud-africaine a élaboré une méthode de justiciabilité des droits économiques et sociaux que plusieurs considèrent comme une étant inspirée du droit administratif, en vertu de laquelle le rôle du tribunal est de vérifier si les politiques publiques sont cohérentes et rationnelles. Traditionnellement, en droit administratif, on oppose l'hypothèse dans laquelle le contrôle est normal parce que la compétence de l'administration serait liée à celle d'un contrôle restreint dans laquelle la compétence ne le serait pas. On ajoute enfin à un troisième cas de figure dans lequel le pouvoir discrétionnaire mis en œuvre devrait faire l'objet d'un contrôle maximum (contrôle de proportionnalité) en raison du domaine qu'il touche, celui des droits et libertés.

Toutefois, on connaît aussi le caractère incertain de ces distinctions qui reposent essentiellement sur des tentatives de systématisations qui ne rendent pas suffisamment compte du rôle essentiel du juge dans la décision de considérer qu'il se trouve bien face à un pouvoir discrétionnaire. Cette interprétation a des conséquences pourtant déterminantes: elle peut laisser penser que l'autorité compétente, ici le législateur, est dotée d'un "simple» pouvoir discrétionnaire, ce qui justifie du même coup un contrôle restreint. En revanche, lorsque le pouvoir mis en œuvre est supposé lié (ce qui pose de redoutable difficulté de démonstration dans le domaine des droits sociaux) parce que tenu de respecter des droits sociaux au contenu déterminé, un contrôle maximum s'imposerait.

1) Contrôle restreint: l'indice d'une intervention législative dans un domaine de " non droits-sociaux"?

Cette hypothèse concerne les cas où le pouvoir discrétionnaire du législateur s'exprime par la mise en œuvre de " politiques sociales » qui mettent en balance droits fondamentaux, notamment droits civils et politiques et "intérêts sociaux", ou encore des "objectifs à valeur constitutionnels" ou encore des " exigences constitutionnelles", selon les termes que l'on trouve dans la jurisprudence du Conseil constitutionnel français. Dans ce cas, le pouvoir discrétionnaire du législateur ne serait pas limité par un contenu prédéterminé d'un droit social : il devrait plutôt mettre en œuvre les moyens de réaliser un objectif, un programme, sans remettre en cause les droits fondamentaux. Le juge se borne alors à un contrôle de "l'irraisonnabilité", i.e. à l'erreur manifeste d'appréciation, " erreur grossière » que le législateur aurait pu commettre dans

761 Minister of Health c. Treatment Action Campaign, [2002] 5 S. Afr. L.R. 721 (S. Afr. Const. Ct.), par. 2-4, 17, 19, 57, 70, 92-95 et 135 (ci-après Treatment Action Campaign). 
le balancement entre les moyens mis en œuvre pour réaliser l'objectif et les droits civils et politiques concernés.

Ainsi, en France, dans la décision du Conseil Constitutionnel 2004-504 sur la loi relative à l'assurance maladie, le principe de protection de la vie privée a été confronté à celui de la protection de la santé publique : le Conseil a conclu à une conciliation qui n'apparaissait pas " manifestement déséquilibrée. "

\section{2) Contrôle normal et/ou maximum: les techniques palliatives de la carence législative}

Ce type de contrôle postule pour le juge l'existence d'un droit social, et précisément d'un contenu normatif. C'est à cette condition que le juge peut dénoncer et sanctionner la carence du législateur.

La Cour constitutionnelle italienne a inventé des formes très variées de décisions - interprétatives, "manipulatives" pour pallier cette carence. Elles vont de la recommandation formulée au législateur à la sanction de la mise en œuvre partielle inconstitutionnelle d'un droit social (215/1987), en passant par l'admission provisoire de la constitutionnalité d'un dispositif (826/1988). Parmi cet éventail non exhaustif, on a déjà parlé des décisions "additives de prestation ". Coûteuses pour le budget de l'Etat, elles ont été très critiquées et ont progressivement laissé la place à des décisions additives "de principe ", jugées plus respectueuses du pouvoir discrétionnaire du législateur. Si la naissance de cette technique est associée à l'exigence de respect du pouvoir discrétionnaire du législateur pour la détermination des ressources financières disponibles, le juge n'a pas pour autant renoncé à la définition du droit social. Par cette technique, il déclare l'inconstitutionnalité de la loi là où elle ne prévoit pas quelque chose qu'elle aurait du prévoir, mais au lieu d'ajouter la règle qui fait défaut, il ajoute un principe dont le législateur devra s'inspirer pour que sa législation soit conforme à la constitution, mais dont le juge a quo peut également faire usage pour résoudre le cas qui lui est soumis. En cela, elle est vue comme une solution qui n'est pas "auto applicative»762. En 1988 (arrêt $n^{\circ} 497$ ) la Cour fournissait une sorte de mode d'emploi de ce type de décision : " la déclaration d'inconstitutionnalité d'une omission législative (...) alors qu'elle laisse au législateur, dont on reconnaît l'indéniable compétence pour introduire et réglementer des mécanismes de protection, même rétroactivement au moyen de normes abstraites, propose elle-même, dans l'attente de l'intervention

\footnotetext{
762 Il existe au Canada une sanction similaire, soit le jugement déclaratoire. Les juges y ont recours lorsque l'interprétation d'un droit fondamental n'est pas encore bien établie mais le devient dans un litige à l'issu duquel il est déclaré que l'État n'a pas respecté le droit en question. Dans ce contexte, les tribunaux déclarent ou constatent, un peu symboliquement, la violation du droit tout en laissant au législateur le soins d'apporter la solution qu'il estime appropriée afin de se conformer à la constitution. Au Canada, dans le domaine des droits et libertés, la Cour suprême a notamment utilisé cette sanction dans le contexte du seul véritable droit "positif " ou droit "créance" reconnu dans la Charte canadienne, soit le droit à l'instruction dans la langue de la minorité francophone ou anglophone dans une province donnée. V. Mahe c. Alberta, [1990] 1 R.C.S. 342 (Cour suprême du Canada).
} 
législative, un principe auquel le juge de droit commun est habilité à faire référence pour remédier à la carence de règle par la voie de la solution du cas individuel et concret qui lui est présenté.». Deux techniques sont donc ici combinées: le recours à un principe formulé par le juge et le contrôle de raisonnabilité sur le fondement de ce principe. Dans ce dispositif, l'alliance entre les juges ordinaires et le juge constitutionnel joue un rôle essentiel. Pour C. Colapietro, cette technique a été vue comme une tentative du juge constitutionnel de poursuivre - à travers la formulation de conditions d'exercice $\mathrm{du}$ pouvoir discrétionnaire du législateur une politique extensive des droits sociaux, en s'érigeant ainsi comme l'unique défenseur de cet Etat social mis en danger, non seulement par une grave crise économique mais également par une politique législative caractérisée par des coupes dans les dépenses qui s'appliquent au premier chef au détriment des conquêtes sociales perpétuées pendant des années ${ }^{763}$.

Dans la mesure où la jurisprudence de la Cour constitutionnelle sudafricaine n'en est encore qu'à ses premiers balbutiements, ses types d'interventions ne sont pas aussi variés que ceux de la Cour constitutionnelle italienne. Jusqu'à présent, bien que la Constitution accorde un large pouvoir de sanction aux juges afin de remédier aux violations des droits économiques et sociaux ${ }^{764}$, la Cour s'est surtout contentée de déclarer ou constater les cas de violation de ces droits et à faire des recommandations assez générales au gouvernement. Ainsi, la Cour n'a à ce jour jamais utilisé le pouvoir dont elle dispose d'ordonner juridiquement au gouvernement la mise en place de mesures plus précises et ni son pouvoir de surveiller le respect de ses jugements par des ordonnances de rendre compte, lesquelles exigeraient que le gouvernement fasse part à la Cour, dans un certains délai, des avancées ou des ratés dans l'élaboration de politiques respectueuses de la Constitution.

\section{Les motifs formulés au soutien des techniques de protection}

Afin de justifier le contrôle du pouvoir discrétionnaire à travers les différentes techniques à peine évoquées les juridictions fournissent des motifs qui doivent être analysés. Parmi ceux-ci, le recours à l'argument d'une prétendue prédétermination des droits sociaux se singularise dans la mesure où la "nature juridique» est bien loin d'être constante et exempte de contradictions(A). La prétendue incompétence institutionnelle et illégitimité politique des tribunaux à se prononcer sur les questions socioéconomiques (B) ainsi que «l'obscur motif de la raisonnabilité»(C) retiendront successivement notre attention.

763 Carlo COLAPIETRO, "Garanzia e promozione dei diritti sociali nella più recente giurisprudenza costituzionale », Giu. It., 1995 p. 125.

${ }^{764}$ Constitution sud-africaine, par. 172 (1) ; Treatment Action Campaign, supra, par. 101-102. 


\section{A. Une prétendue prédétermination des droits sociaux}

1) "Droits subjectifs et inviolables": des qualifications favorables à la protection

La qualification de droit subjectif permet l'économie d'un discours justificatif: l'applicabilité directe des droits sociaux est présentée comme une conséquence de cette "nature juridique». La Cour constitutionnelle italienne dans un arrêt de 1960 n$^{\circ} 30$ affirme que l'article 36 de la constitution énonce un droit subjectif parfait sans renvoi à la loi ordinaire. La qualification de droit fondamental de la personne, très présente dans la jurisprudence récente semble bien jouer le même rôle que la notion de droit subjectif. Ainsi, dans l'arrêt n ${ }^{\circ} 252$ de 2001 la Cour affirme que : "Le 'noyau irréductible' de protection de la santé en tant que droit fondamental de la personne doit être reconnu également aux étrangers, quelle que soit leur position par rapport aux normes qui règlent l'entrée et le séjour dans l'Etat, et même si le législateur peut prévoir différentes modalités d'exercice de celui-ci. »

L'argument de l'inviolabilité des droits est quant à lui invoqué notamment pour justifier la non rétrogression du droit en dessous d'un contenu minimum essentiel ainsi que pour isoler un noyau qui serait même inviolable par le législateur constituant. Les droits sociaux à eux seuls, ou associés au principe de dignité (voir supra) sont à plusieurs reprises qualifiés directement par le juge de droits inviolables et irrétractables de la personne, en tant qu'ils expriment des valeurs ou des principes constitutionnels suprêmes: il en est ainsi, parmi d'autres, du droit à la santé (1011/1998, 294 et 184/1986, 88/1979), du droit au logement (19/1994, 404 et 217/ 1998), du droit au travail (108/1994 et 232 /1989).

\section{2) Des qualifications moins favorables}

\section{a. Des droits conditionnés par les ressources financières}

La jurisprudence italienne en matière de droits sociaux a été considérablement marquée par une décision de 1990 (455/1990) relative au droit à la santé dans laquelle a été affirmé le caractère "conditionné " des droits sociaux. En effet, elle semble rétablir la distinction entre disposition programmatique et les dispositions prescriptives que la Cour avait pourtant écarté dès sa première décision. Elle scinde le droit à la santé en un droit défensif et un droit social, le droit à recevoir des prestations sanitaires : au sujet de ce dernier, elle observe que le droit à la santé est " un droit constitutionnel conditionné dont la réalisation dépend du législateur en tenant compte des limites objectives que ce même législateur rencontre dans son action de mise en œuvre de la constitution en relation avec les ressources institutionnelles et financières dont il dispose à ce moment". La Cour y affirme encore que la réalisation des droits sociaux doit tenir compte également d'un "raisonnable balancement avec d'autres intérêts ou biens qui jouissent de la même façon de 
protection constitutionnelle " et que ce "balancement " serait " lui aussi l'objet d'un contrôle de la part de la Cour, dans les formes et dans les modes appropriés à l'usage du pouvoir discrétionnaire du législateur". Enfin, dans une décision n 417/1996 la Cour rejette une question de constitutionnalité et affirme qu'« il n'est pas possible d'exclure la possibilité d'une intervention législative qui, en raison de l'indérogeable exigence de limitation de la dépense publique, réduise définitivement un traitement de pension précédemment prévu, étant donné qu'il existe la limite des ressources disponibles".

Au Canada, les tribunaux s'appuient aussi le caractère "conditionné " des droits économiques et sociaux pour en réduire la portée juridique ou tout simplement refuser de reconnaître leur caractère fondamental et constitutionnel et les restreindre à de simples déclarations d'intentions politiques. Par exemple, dans l'arrêt Gosselin en première instance, la Cour supérieure affirmait : " Il y a une différence de nature entre d'une part les droits économiques et sociaux qui exigent une intervention active et l'engagement de ressources importantes de l'État pour leur mise en œuvre et d'autre part, les droits civils et politiques qui n'exigent généralement que des aménagements aux institutions politiques et juridiques et sont ainsi susceptibles de mise en œuvre immédiate par les États quelque soit leur niveau de développement ${ }^{765}$ ". La Cour suprême du Canada confirmait cette interprétation en soulignant le caractère programmatoire des droits économiques et sociaux dont la mise en œuvre dépend nécessairement d'une intervention législative et, conséquemment, dont la portée réelle serait déterminée exclusivement et discrétionnairement par le législateur ${ }^{766}$.

\section{b. Des objectifs à valeur constitutionnelle}

Dans la jurisprudence du Conseil constitutionnel français, on retrouve les incertitudes exprimées dans la jurisprudence constitutionnelle italienne : il en est ainsi du droit à la santé et du droit au travail pour lesquels le Conseil utilise les formules de "droit à ", mais réserve un traitement souvent identique à celui qu'il applique aux objectifs à valeur constitutionnelle, notamment en restreignant le contrôle de leur respect à celui de l'erreur manifeste d'appréciation ${ }^{767}$. Il se refuse par la même occasion à formuler des standards jurisprudentiels permettant de cerner les contours des droits sociaux. Par ailleurs, la jurisprudence sur les "garanties légales des exigences constitutionnelles" n'a jamais conduit le juge français à constater la méconnaissance de l'interdiction de priver de garanties légales les exigences

765 Gosselin c. Québec (Procureur général), [1992] R.J.Q. 1647 à la p. 1669 (Cour supérieure du Québec) [Gosselin (Cour supérieure)]. V. également Gosselin (Cour suprême), supra, par. 89, juge McLachlin, 421, juge Le Bel; Gosselin c. Québec (Procureur général), [1999] R.J.Q. 1033 à la p. 1047, juge Baudouin (Cour d'appel du Québec) [Gosselin (Cour d'appel)] ; Lévesque c. Québec (Procureur général), [1988] R.J.Q. 223 (Cour d'appel du Québec) ; Commission scolaire St-Jeansur-Richelieu c. Commission des droits de la personne du Québec, [1994] R.J.Q. 1227 à la p. 1242 (Cour d'appel du Québec).

766 Gosselin (Cour suprême), supra, aux par. 85 à 96 ; Gosselin (Cour d'appel), Ibid., à la p. 1047.

767 Décision 94-359 D.C. du 19 janvier 1995, Loi relative à la diversité de l’habitat. 
constitutionnelles en matière sociale ${ }^{768}$. Cet ensemble d'éléments semble témoigner du retrait du Conseil constitutionnel du domaine de la définition des droits sociaux laissée précisément à l'appréciation du législateur. Enfin, il convient de souligner que le caractère prédéterminé d'un droit social, en l'occurrence le droit de grève, a été établi dans la jurisprudence constitutionnelle française par le recours à l'argument de l'intention du constituant ${ }^{769}$, ce qui pourrait être une voie pour la formulation de standards pour les droits sociaux non défensifs.

\section{c. Des droits qui ne peuvent se réaliser que progressivement 770}

Alors que l'engagement pris par les États parties au PIDESC de réaliser progressivement les DESC en fait clairement une des techniques de protection des droits sociaux ${ }^{771}$, dont on peut comprendre qu'elle entretient des liens étroits avec la notion de contenu minimal essentiel ${ }^{772}$, on doit souligner que ce principe de réalisation progressive a fait l'objet de vives critiques en Italie. Encore une fois il a été vu comme une expression de self restraint du juge. Il est notamment critiqué en raison de la présomption de légitimité qu'il semble conférer aux réglementations qui mettent en œuvre les droits sociaux, excluant d'office les possibilités de censure du caractère partiel de la mise en œuvre, de l'inadéquation de la mesure.

Par opposition à ce principe de " gradualité " vers le bas, une lecture plus positive est également présente dans la jurisprudence et dans la doctrine quand elle correspond à un accroissement progressif et graduel du niveau de garanties, en somme un principe de gradualité vers le haut.

\section{B. Des questions socioéconomiques qui ne relèveraient pas de la compétence des tribunaux}

Au Canada, la stratégie la plus souvent déployée pour dénier aux droits économiques et sociaux le caractère de droits fondamentaux consiste à les opposer aux droits civils et politiques. Alors que le droit à la vie, la liberté d'expression, et d'autres droits et libertés classiques seraient intrinsèques à la nature humaine et n'exigeraient ce faisant aucune intervention étatique particulière ${ }^{773}$, il en irait autrement en ce qui concerne les droits économiques et sociaux. Ceux-ci n'existeraient pas concrètement à l'état originel de " nature » et ne seraient pas universels puisque seuls les individus non méritoires auraient

\footnotetext{
768 CC 86-225 DC 23 janvier 1987.

769 Décision n 2007-556 DC du 16 août 2007.

770 Carmela SALAZAR, Dal riconoscimento alla garanzia dei diritti sociali. Orientamenti e tecniche decisorie della Corte costituzionale a confronto, précit., p. 128.

771 Cette obligation ressort, en droit international, de l'article 2(1) du PIDESC.

${ }_{772}$ Effectivement, l'obligation de l'État ne saurait se limiter à ne protéger que le contenu minimal d'un droit social. Une fois ce niveau atteint, l'État n'en demeure pas moins tenu de continuer à faire des efforts soutenus en vue d'assurer, à terme, une protection plus efficace, voir la pleine réalisation, des droits économiques et sociaux.

${ }^{773}$ Gosselin (Cour supérieure), supra, à la p. 1669.
} 
besoin de leur protection. C'est d'ailleurs la conception qui ressort de la jurisprudence canadienne, alors que la pauvreté semble être considérée comme un problème qui résulte de la "faute " ou des mauvais choix de vie de l'individu qui la subit et qui, conséquemment, a besoin d'une aide de dernier recours de la part de l'État 774 . Les tribunaux doutent par ailleurs de leur compétence à se prononcer sur des questions socioéconomiques complexes ainsi que la légitimité politique pour un juge de remettre en question les choix budgétaires du gouvernement. La réalisation des droits sociaux implique en effet pour l'État la dépense de fonds publics ${ }^{775}$. Considérant la pression que cela exercerait sur les ressources étatiques, les tribunaux canadiens, de même que la Cour constitutionnelle sud-africaine malgré le caractère plus progressiste de sa jurisprudence, considèrent trompeur de promettre aux citoyens, sous la forme de droits fondamentaux, la satisfaction de besoins dont la matérialisation pour tous est impossible ${ }^{776}$. Puisque l'État n'est pas responsable de toutes les inégalités socioéconomiques et qu'il est souverain sur les questions budgétaires, il serait illégitime, au nom de la séparation des pouvoirs, que des juges non élus fassent de la "politique» en dictant au gouvernement comment dépenser son argent 777 .

On soutient par ailleurs que le " contenu obligationnel "778 des droits économiques et sociaux est trop variable ou incertain pour qu'ils soient formulés et garantis dans des instruments juridiques constitutionnels reconnaissant des droits universels. Le droit au logement, par exemple, implique-t-il que l'État doive construire des habitations à loyers modiques ou qu'il subventionne directement les personnes dans le besoin afin qu'elles puissent elles-mêmes se trouver un toit? Aussi, quelles doivent être la qualité et les caractéristiques de l'immeuble qui fait l'objet du droit individuel Voilà le genre de questions spécifiques ou de détails auxquels la fonction judiciaire ne serait pas nécessairement bien placée pour répondre ${ }^{779}$. Les tribunaux estiment ainsi généralement que la branche politique de l'État, par son expertise sociale et économique, est mieux à même d'évaluer les besoins de la population et développer des programmes qui y répondent ${ }^{780}$.

\footnotetext{
${ }^{774}$ Gosselin (Cour supérieure), Ibid. à la p. 1676.

775 Gosselin (Cour d'appel), supra, à la p. 1047, juge BAUDOUIN ; Masse v. Ontario (Ministry of Community \& Social Services), [1996] O.J. No. 363 (Ont. Div. Ct.), aux para. 42-43, juge O’Driscoll, 56, juge O'Brien ; Gosselin (Cour supérieure), supra, à la p. 1669.

776 Government of the Republic of South Africa c. Grootboom, [2001] 1 S. Afr. L.R. 46 (Cour constitutionnelle sud-africaine), au par. 35.

777 V. Masse, supra, par. 43, juge O'Driscoll, 58, juge O'Brien ; Clark v. Peterborough Utilities Commission, (1995), 24 O.R. (3d) 7 [Div. gén. Ont.], par. 28 ; Conrad v. Halifax (County) et al., (1994), 124 N.S.R. 251 [Cour supérieure de la Nouvelle-Écosse], par. 93, 95 ; Gosselin (Cour supérieure), supra, aux pp. 1669-1670.

778 Nous empruntons cette expression au juge LeBel : Gosselin (Canada, C.S.), supra, par. 429.

779 Grootboom, supra, par. 32, 37 et 38.

780 Clark, supra, par. 28 ; Conrad, supra, par. 93, 95.
} 


\section{La "raisonnabilité " des choix législatifs: un motif certainement obscur, et donc réellement efficace ?}

Le caractère insaisissable de l'argument de la raisonnabilité du choix du législateur pose évidemment le problème du pouvoir considérable qu'il remet entre les mains du juge. Certaines tentatives doctrinales reprises par la jurisprudence en Italie ont tenté en dépit des ambigüités des termes, de désamorcer les critiques en soutenant le caractère "lié " de ces jugements de "rationalité » (1). Mais si l'on admet que la raisonnabilité est une justification qui suppose une appréciation en termes de valeurs, il peut être intéressant d'observer alors quels sont les «bons" arguments retenus dans la jurisprudence. L'efficacité de l'argument n'en sera que plus évident : toutefois celle-ci n'est pas toujours favorable aux droits sociaux (2).

1) "Raisonnabilité »/« rationalité ": une distinction doctrinale et jurisprudentielle pour masquer le pouvoir des juges

Il est apparemment facile d'expliquer le recours à l'argument de la raisonnabilité en rappelant que les droits sociaux interviennent dans un domaine où le législateur dispose dans leur mise en œuvre d'un pouvoir discrétionnaire. Aussi, pourrait-on dire que la solution ne peut se déduire logiquement des dispositions constitutionnelles, d'où le recours à un contrôle de raisonnabilité, c'est-à-dire d'adéquation entre les moyens et les fins.

L'argument de la raisonnabilité au sens de rationalité (raisonnabilitérationalité) n'est qu'une autre formulation de l'argument de la compétence liée du législateur. Il a été mobilisé, et cela n'est guère surprenant, pour légitimer les décisions additives de prestations, i.e celles qui sont le plus irrespectueuses du pouvoir discrétionnaire du législateur. Il se construit sur l'affirmation du caractère univoque des dispositions utilisées pour combler l'omission législative, que l'expression doctrinale et jurisprudentielle de décision "à rimes obligées" illustre parfaitement.

A coté de cette première stratégie qui consiste à nier le pouvoir du juge, on trouve une argumentation plus moderne, mise en œuvre au soutien des additives de principe. Il n'est plus question de déduction ici, mais d'une appréciation de l'adéquation (de l'opportunité) d'un choix de moyens pour la réalisation d'une fin. La norme qui sert de paramètre pour le contrôle de constitutionnalité laisse le choix des solutions au législateur, la norme serait muette selon le juge. Mais le juge dégage de l'ensemble des valeurs consacrées par le système juridique un principe constitutionnel: quand la Cour trouve une lacune qui pour être comblée nécessiterait un choix normatif de type politique, elle ne peut pas émettre une décision "à rimes obligées", étant donné la pluralité d'options normatives qui pourraient se présenter pour remplir le vide laissé par le législateur. En l'espèce, au lieu d'"imposer" une solution et donc d'exercer de manière impropre une fonction de législation positive, la Cour "découvre" un principe, au moyen duquel elle ne fait pas autre chose que de 
définir le droit social, et en impose le respect au législateur et au juge a quo pour le cas qui lui est soumis.

2) La " raisonnabilité " du choix législatif : les "bons" arguments selon le juge constitutionnel

Selon R. Guastini l'argument de la raisonnabilité est un argument tautologique. Les tribunaux, on l'a vu, ont forgé le principe de raisonnabilité sur le fondement d'une interprétation désormais répandue du principe d'égalité selon laquelle: les cas égaux doivent être traités de façon identique, les cas différents de façons différentes: "Mais comment décider quels sont les cas semblables et quels sont les cas différents? En d'autres termes, quelles sont les distinctions justifiées et quelles sont les distinctions constitutives de discriminations ? Et bien, le principe de raisonnabilité constitue précisément une réponse à cette question. Malheureusement, il s'agit d'une réponse tautologique. Selon ce principe, les distinctions (ou classifications) opérées par le législateur doivent être raisonnables pour ne pas être discriminatoires. Qu'est-ce que cela veut dire? Cela signifie que, pour distinguer, il faut qu'il y ait des raisons, naturellement. Mais que faut-il entendre par 'raisons' ? Dans ce type de contexte, il est évident que 'raisons' ne signifie pas simplement argument : il signifie «bons » arguments, c'est-à-dire des justifications. Donc, selon le principe de raisonnabilité, une distinction est justifiée quand elle est justifiée » ${ }^{781}$.

Cette critique de l'auteur italien peut être ici utilisée pour examiner les "bons" arguments que le juge retient dans le domaine des droits sociaux. Décider qu'un argument est "bon", qu'un choix législatif est justifié, c'est naturellement porter sur lui un jugement de valeur. Le jugement de raisonnabilité confère en principe au juge un instrument très puissant pour revoir les choix discrétionnaires du législateur (b). Encore faut-il toutefois qu'il ait décidé de faire usage de cet instrument. Dans le cas contraire, c'est une politique jurisprudentielle de self-restraint qui s'exprime lorsque, selon le juge, la compétence discrétionnaire du législateur en matière d'équilibre budgétaires est en jeu (a).

\section{a. La raisonnabilité " en soi " du respect de l'équilibre budgétaire}

Le caractère raisonnable d'un choix ou d'un balancement entre des principes réalisés par le législateur peut venir justifier la restriction du contrôle (voir supra). Cette restriction manifeste de la part du juge une présomption implicite de faveur pour le principe du pouvoir discrétionnaire du législateur, c'est notamment le cas lorsque l'argument du respect de l'équilibre budgétaire est mobilisé.

\footnotetext{
781 Riccardo GUASTINI, « La costituzione come limite alla legislazione», Analisi e diritto, 1998,
} disponibile en ligne, www.giuri.unige.it/intro/dipist/digita/filo/.../Guastini1.pdf. 
ou même l'étendre, il justifiera sa préférence en renforçant son poids dans la balance face au principe du pouvoir discrétionnaire du législateur (décision 309/1999 au principe de dignité) ${ }^{784}$.

Enfin, si l'argument de la raisonnabilité/égalité permet de justifier des politiques jurisprudentielles très audacieuses et pénétrantes dans la sphère de décision du législateur, notamment au moyen des techniques de sentences additives de prestations, le caractère minimal/essentiel du droit ne semble pas compatible avec une exigence de "raisonnabilité/égalité", selon laquelle tout avantage conféré par la mise en œuvre d'un droit social devrait être étendu à des catégories qui en seraient illégitimement exclues.

Au contraire, le contenu minimal essentiel, pris isolément, justifie la limitation de l'extension des garanties dans le sens d'une réduction ou égalisation vers le bas et non vers le haut. Combiné cependant au principe de progression reconnu en droit international, il oblige le gouvernement, une fois réalisé le contenu minimal ou essentiel des droits sociaux, à poursuivre ses efforts en vue d'assurer éventuellement la pleine réalisation de ses droits et, conséquemment, une meilleure qualité de vie aux citoyens.

Pour citer cet article

Isabelle Boucobza et David Robitaille, «Standards jurisprudentiels et contrôle de l'obligation étatique en droit comparé : une géométrie variable », La Revue des Droits de l'Homme, juin 2012 http://revdh.files.wordpress.com/2012/06/standards-jurisprudentiels-et-contrc3b4le-de-1_obligationc3a9tatique-en-droit-comparc3a9-une-gc3a9omc3a9trie-variable.pdf

\footnotetext{
784 « La protection du droit à la santé ne peut pas subir les mêmes contraintes que le législateur rencontre dans la distribution des ressources financières dont il dispose ". La Cour a en effet affirmé de façon répétée (sent. nn. 267 de 1998, 416 de 1995, 304 e 218 de 1994, 247 de 1992, 455 de 1990) que les exigences des finances publiques ne peuvent assumer dans le balancement du législateur, un poids si prépondérant qu'il conduise à comprimer le noyau essentiel du droit à la santé protégé par la constitution en tant que domaine inviolable de la dignité humaine».
} 